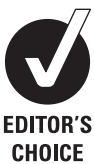

CHOICE

\title{
Age, comorbidity and functional status influence end-of-life decisions in critical illness, while nationality, ethnicity and clinician experience influence the agressiveness of medical care
}

\section{Ronald L Hickman, Jr}

\subsection{6/ebnurs.2011.100275}

Frances Payne Bolton School of Nursing, Case Western Reserve University, Cleveland, Ohio, USA

Correspondence to:

Ronald L Hickman, Jr

Case Western Reserve

University, Frances Payne Bolton

School of Nursing, 2120 Cornell

Road, Cleveland, $\mathrm{OH}$ 44106-

4904, USA;

ronald.hickman@case.edu

\section{Commentary on: Frost DW, Cook DJ, Heyland DK, et al. Patient and healthcare professional factors influencing end-of-life decision-making during critical illness: a systematic review. Crit Care Med 2011;39:1174-89.}

\section{Implications for practice}

- Critical care nurses are more likely to recommend intensive end-of-life (EOL) care compared with physicians who routinely work in critical care settings.

- Patients with advanced age, comorbidity and limited functional status are less likely to use technologically intensive EOL care.

\section{Implications for research}

- Further evaluation of the complex interactions of factors associated with critically ill patients, family members and healthcare professionals are needed to guide the development of decision support strategies at EOL.

- Novel decision support strategies should consider tailored approaches to overcome patient and healthcare professional factors and facilitate informed decisionmaking at EOL.

\section{Context}

Despite the intense use of technology and aggressive medical care, intensive care units are common places of EOL decision-making and death. ${ }^{1-3}$ Most critically ill patients are unable to participate in EOL decision-making due to the severity of their illness or need for sedation. In an effort to respect the ethical principle of autonomy, healthcare professionals engage the patient's family in the EOL decision-making. ${ }^{45}$ Although there is a growing body of literature on EOL decision-making during critical illness, there has been limited synthesis of these findings to guide clinical practice and research. The systematic review (SR) conducted by Frost et al identifies several factors associated with patients and healthcare professionals that influence EOL decision-making.

\section{Methods}

The authors conducted a SR to reveal the influential factors of critically ill patients and healthcare professionals that influence EOL decision-making. They defined EOL decision-making as "any decision related to choices for care provided to currently or potentially critically ill patients'. Publications included were controlled trials, cohort and survey studies published in English which explored the associations among patient or healthcare professional characteristics and adult EOL decision-making. Of 6259 publications identified, 102 publications met the inclusion criteria. Due to the heterogeneity of the publications meta-analytic techniques were not conducted. However, qualitative descriptions of each factor that influenced EOL decision-making are provided.

\section{Findings}

The patient's age, comorbidity and functional status, gender and race/ethnicity were identified as the most frequent factors that influence the technological intensity of EOL care in critically ill patients. Patients with increasing age, comorbidity and limited functional status were less likely to use technologically intense EOL care. Less-frequently examined patient-level factors (diagnosis, religiosity, personality traits and geographic location) were associated with EOL decision-making. In contrast, the specialty, years of clinical experience and place of training of healthcare professionals were the most frequent characteristics found in this SR to have an influence on EOL decision-making. Experienced critical care physicians are less likely to endorse technologically intense care at EOL for critically ill patients.

\section{Commentary}

This is a well-constructed study of patient and healthcare professional factors that influence EOL decision-making. Frost et al employed recommended search strategies, conducted quality assessments of each identified publication, and clearly defined how the inclusion criteria for the 102 publications were used to inform their findings. Despite the implementation of highly rigorous methods for an SR, the authors made a significant trade-off to enhance the generalisability of their findings by including studies of non-critically ill patients and healthcare professionals who did not routinely work in critical care settings. As noted by the authors, this decision to include 'potentially' critically ill patients and non-critical care healthcare professionals contributed to heterogeneity of studies included in the SR, but precluded the conduct of meta-analytic techniques. With this, the authors were limited to reporting frequency 
data and qualitative descriptions of patient and healthcare factors associated with EOL decision-making.

Frost et al acknowledged several limitations in this study. All non-English publications were excluded; several unpublished studies were missed and, heterogeneity of studies prevented the use meta-analytic techniques and resulted in a qualitative summary of findings.

\section{Competing interests None.}

\section{References}

1. Bryce C, Loewenstein G, Arnold R, et al. Quality of death: assessing the importance placed on end-of-life treatment in the intensive care unit. Med Care 2004;42:423-31.
2. Levy MM, Rapoport J, Lemeshow S, et al. Association between critical care physician management and patient mortality in the intensive care unit. Ann Intern Med 2008;148:801-9.

3. Goldhill DR, Sumner A. Outcome of intensive care patients in a group of British intensive care units. Crit Care Med 1998;26:1337-45.

4. Hickman R, Daly B, Douglas S, et al. Informational coping style and depressive symptoms in family decision makers of the cognitively impaired chronically critically ill. Am J Crit Care 2010;19(5):410-20.

5. Hickman RL Jr, Douglas SL. Impact of chronic critical illness on the psychological outcomes of family members. AACN Adv Crit Care 2010;21:80-91. 